\title{
"Interactive Spatial Copy Wall" for embodied interaction in a virtual co-existing space
}

\author{
*Shigeru WESUGI, **Kazuaki ISHIKAWA, **Nobuyoshi SUZUKI, *Yoshiyuki MIWA \\ *School of Science and Engineering ** Graduate school of Science and Engineering \\ Waseda University \\ 59-319, 3-4-1, Okubo, Shinjuku, \\ Tokyo, 169-8555, JAPAN \\ Waseda University \\ 59-319, 3-4-1, Okubo, Shinjuku, \\ Tokyo, 169-8555, JAPAN \\ E-mailwesugi@computer.org, \{kaputt@suou.,n-suzuki@asagi.,miwa@\}waseda.jp
}

\begin{abstract}
A significance of a sense of togetherness or co-existence among remote communication partners has been pointed out in computer mediated communication area. In order to create such a co-existing situation, the presence of remote partners should be expressed at each place. In this paper, we propose a novel tangible human avatar display for remote interpersonal communication based on an idea integrating a video avatar expressing the fidelity of the embodiment, with a robot avatar as the physical presence. As a result, we devised "Interactive Spatial Copy Wall" ("ISCW")system which represents a three-dimensional shape of remote partners with hundreds of movable pipes and remote participants can interact with each other by operating these movable pipes. We design this system based on modularity and we construct "ISCW"system of 192 cylinder modules. At the end we discuss a possibility of this tangible avatar display when compared to the previous communication systems.
\end{abstract}

\section{Introduction}

For these decades, numerous telecommunication systems have been developed and proposed. These systems can support remote awareness - for example, who are participating, what they are doing, and where they are by expressing bodily actions of a remote partner such as gaze and gesture [1]. A problem of disembodiment, however, occurs in such computer-mediated communication, as Dreyfus pointed out [2]. For example, even when life-sized image of a remote partner appears on a large screen at a local place, psychological distance to the person in the screen differs from that in a face-to-face communication situation [3]. We postulated that presence of a remote partner locates itself just in a screen or behind a screen not in the face-to-face situation, where, in contrast, people are bodily present at the same place. This fullbodied presence is crucial to everyday human encounters [2]. We can attune ourselves to mood, when we are bodily present in a situation. The situation - we are embedded with others and things in a common setting - is called as "Ba" in Japanese [4][5]. "Ba" is not a physical place but implies a co-existing situation in mind. We believe that creation of co-existing situation is fundamental to precede interpersonal communication [4][5]. Here we use a word "co-existing space" as a common place at which people are full-bodied present.

A requirement of communication technology creating a co-existing space between remote places will increase more than ever, since a few communication systems have been proposed and received attention to support a sense of connectedness among remote families and a remote watching an elderly person living alone peacefully in several years [6][7]. In order to address this challenge, we have paid attention to enhance a sense of "interconnectedness" among remote people in a shared space and implemented several communication systems [8][9]. Consequently, we found our systems possess capability to enhance a sense of co-existence between remote participants.

This paper describes subsequent steps of developing this design method for creating a virtual co-existing space in a physical place where our body exists. The paper discusses a concept and design process of a novel tangible human avatar display for remote interpersonal communication which is based on an idea integrating a video avatar expressing the fidelity of the embodiment, and a robot avatar as the physical presence. As a result, we devised "Interactive Spatial Copy Wall" system which represents a three-dimensional shape of remote partners with hundreds of movable pipes and remote participants can interact with each other by operating these movable pipes. At the end, we discuss a possibility and uniqueness of this tangible avatar display when compared to the previous communication systems.

\section{Design of a virtual co-existing space}

There are few design frameworks and approaches to create a virtual co-existing space, since most of previous communication systems are intended to support primarily how to convey bodily action such as gaze awareness [10][11] and gesture in a shared workspace [11][12][13]. Creation of a co-existing space is considered based on interconnectedness between space and self, and between the other communication partner and self simultaneously [4][5]. As a result we proposed an idea to enhance such interconnectedness by focusing on interconnected relationship between self and a tool when using the tool. 


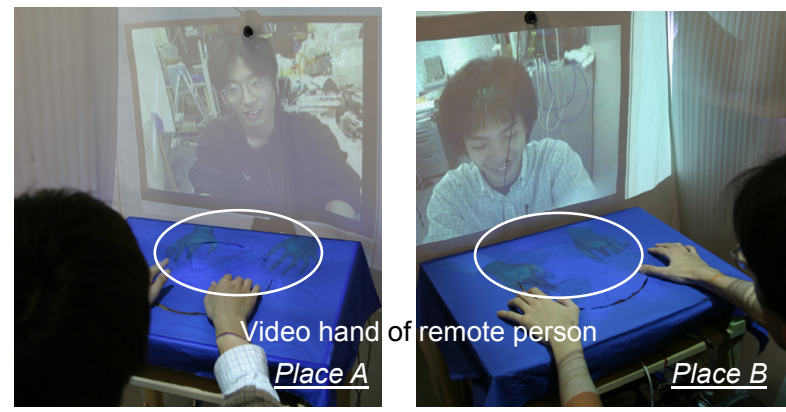

Figure 1. "LazySusan" communication system

Then we devised a networked "Lazy Susan" communication system which integrates tangible interaction with physical disk -the rotation of which is synchronized with that of remote disk- and visual interaction in a shared virtual environment - representing both avatars interacting with each other- [8]. Consequently, we found our system could enhance a sense of coexistence in a shared virtual space[9]. Additionally, we developed this design method for creating a virtual coexisting space in a physical place where our body exists. As a result we implemented a novel video projection "Lazy Susan" communication system which represents reflection of self in a common video space, and projects reflection of a remote partner onto a local tabletop, and remote participants can interact with each other by rotating a networked disk as shown in Figure 1[10]. The results of experiments suggest our system can enhance interconnectedness between self and remote place, and between remote partner and local place, and simultaneously enhance a sense of co-existence. These our communication systems propose a design method of integrating representing reflections of communication partner and self, and interacting with a physical object that is linked with a corresponding remote one, in order to support a sense of co-existence. We intend to create a virtual co-existing space where people can do much more flexible spatial embodied interaction than in our previous systems based on this idea, and devise an idea of "Interactive Spatial Copy Wall"("ISCW"). Our idea of "ISCW" comes from a toy of pin display in Figure 2. This toy can represent a three-dimensional shape of a physical object by pushing the object onto hundreds of pins from behind. We apply this idea for representing remote participants and remote objects as shown in Figure 2.

\section{DESIGN OF SYSTEM}

\section{Mockup of "Interactive Spatial Copy Wall"}

At the beginning we built a full-scale mockup wall in order to investigate how people use our communication system, and to consider specification of a display on whole size, size of pin, number of pin, and arrangement of pins. Cylinders and wall of mockup are made of styrene foam because of easy work, lightness and cost. After each part is cut and sanded, all parts are coated with resin for

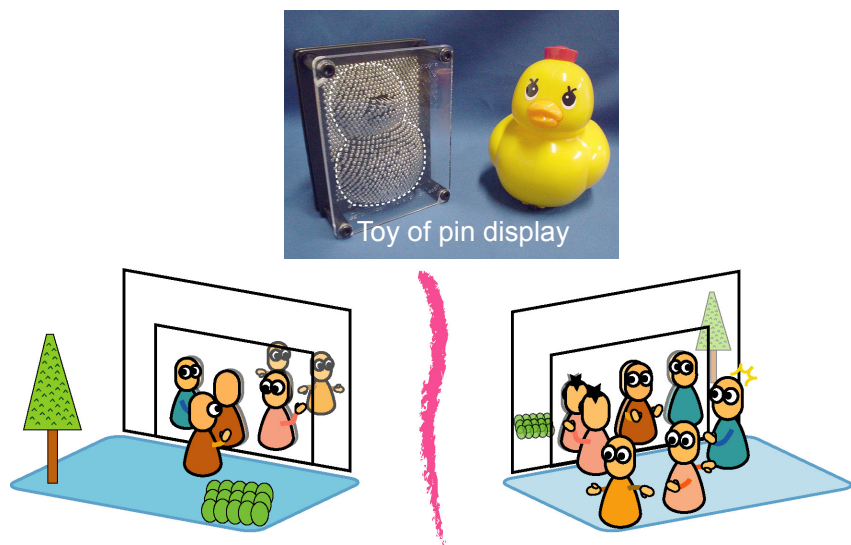

Figure 2. Design of "Interactive Spatial Copy Wall"

increasing durability. This mockup (900x920x210 [mm]) includes approximately 500 cylinders, each of which is arranged horizontally by $30[\mathrm{~mm}]$ and vertically by 40 [mm]. Each cylinder can be transformed manually from 0 to approximately $300[\mathrm{~mm}]$. Additionally video of remote participant can be projected onto the surface of the wall. We observed how people moved these cylinders and what they expressed with these cylinders. The results suggest that people use our mockup in two interaction modes as shown in Figure 3.

1. Displaying mode

- The surface of the wall can represent an appearance of remote participants.

- The surface of the wall can transform according to bodily actions of remote participants.

- The surface of the wall can also represent an appearance of self when close to the wall.

2. Haptic interaction mode

- Local participants can interact with remote participants through pushing and pulling cylinders.

- Both participants can model a shape with cylinders collaboratively.

We obtained system requirements: wall size $500 \times 700[\mathrm{~mm}]$, approximately 200 cylinders $(30[\mathrm{~mm}]$ in diameter), arranging cylinders by $40[\mathrm{~mm}]$, cylinder stroke from 0 to $250[\mathrm{~mm}]$.

Pilot system of "Interactive Spatial Copy Board"

Subsequently, we implement "Interactive Spatial Copy Board" ("ISCB") system as a part of full-scale system, which includes a board $(300 \times 400[\mathrm{~mm}])$ and 32 pipes $(30[\mathrm{~mm}]$ in diameter). Each pipe moves from 0 to $100[\mathrm{~mm}]$. This "ISCB" system is networked with another system via LAN, and each pipe moves synchronously with a remote corresponding one as shown in Figure 3. We tried to integrate these multiple "ISCB" units in order to construct full-scale "Interactive Spatial Copy Wall" system, however, each pipe cannot moves much over $100[\mathrm{~mm}]$ because this unit apply crank shaft mechanism for moving a pipe. In order to make cylinder move much 


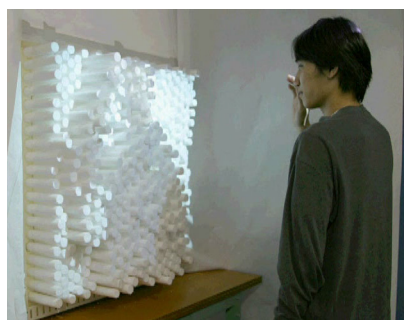

(a) Displaying mode

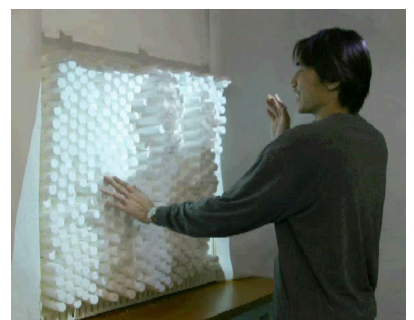

(b) Haptic interaction mode
Figure 3. Utilization of mockup

more in this mechanism, rotating rod should become long, and space for rotation also should be required. This means the unit cannot be downsized in principle. Additionally, 32 cylinder drive mechanisms are intricately incorporated in this "ISCB" unit, so that whole unit should be disassembled in order to adjust and modify one drive mechanism.

\section{Design of "Interactive Spatial Copy Wall"}

The "ISCW" system should realize to control and sense motion of hundreds of pipes in real time. The system requires an inventive idea especially from the view of mechanism and data transmission. We design the "ISCW" system based on modularity. One cylinder module is the smallest element of "ISCW" system, such as a pixel in a picture, and includes one mechanism to move a pipe and a control board for data transmission. Multiple these units can construct "ISCW" system. One pipe $(30[\mathrm{~mm}]$ in diameter as well as in mockup and "ISCB" unit) should move at least $250[\mathrm{~mm}]$ in order to represent thickness of head and upper body. A pipe drive mechanism should be simple and downsized. A case holding a drive mechanism and a circuit board in side should be connected tightly and simply with other cases to construct a "Wall".

Additionally, each cylinder module should transmit data to remote corresponding module, and multiple pipes also should move at once in real time. Algorithm of transmitting ID of module and position data of its pipe between $\mathrm{PC}$ and each module, and between $\mathrm{PC}$ and remote PC should be invented and implemented in order to control hundreds of cylinder module. There are bottlenecks with increasing number of module; load in communicating process between PC and each module, and number of ID attached to each module. In order to solve these problems, we devise an approach;

1. 16 cylinder modules construct one controller unit.

2. PC can communicate with each controller unit.

This approach will remove complication that PC communicates with all cylinder modules, and avoid proportional increase communicating process with increasing number of cylinder module. Additionally, 16 cylinder-module IDs are enough because PC can just communicate with several controller units of 16 modules. Even when number of cylinder module increases, number

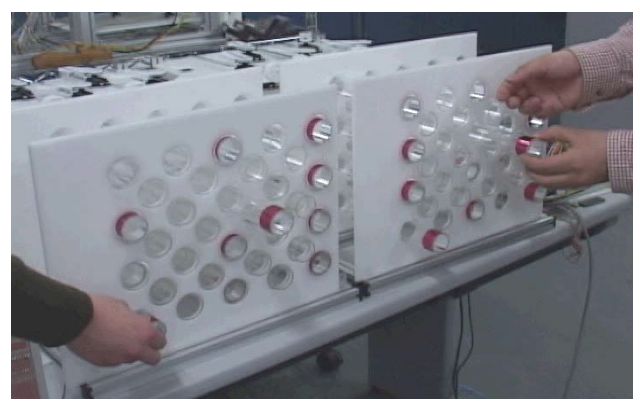

Figure 4. "Interactive Spatial Copy Board"

of controller unit just increases. This will suppress effects of proportional increase number of cylinder module.

Additionally, for displaying mode, three dimensional figure of people in front of system should be measured non-contactly.

\section{IMPLEMENTATION}

\section{Cylinder module}

One cylinder module includes inside one mechanism to move a pipe and one control board for data transmission in a cuboid $(42 \times 39 \times 470[\mathrm{~mm}])$. The pipe drive mechanism has one DC motor and one rotary encoder inside, and controls a motion of cylinder on a rack-and-pinion mechanism which can realize a downsizing- cross section area of cylinder module case is approximate to that of pipe $(30[\mathrm{~mm}]$ in diameter $)-$, a long moving distance $(250[\mathrm{~mm}])$ and a smooth interaction $(2.9[\mathrm{~N}])$. The control board is composed of microcomputer (PIC16F74), rotary encoder counter and motor driver. The microcomputer calculates PID parameter from the encoder counter data and the targeted value sent from PC and transmits PWM signal to control position of pipe. Then we implement a simulation board to check whether each cylinder module can work adequately by testing performance of pipe movement and data transmission from PC. A case of cylinder module can connect with another case around itself with two joints; front joint fitting with each other such as a piece of jigsaw puzzle, and back joint hooking each case with a pin.

\section{Data communication}

Two-stage controller units are installed between PC and cylinder as shown in Figure 6. An upper stage controller unit can control a traffic between PC and lower stage controller unit, and lower stage controller unit can control 16 cylinder modules. Each controller unit includes microcomputer (PIC16F877) inside.

In down stream from $\mathrm{PC}$ to module, the upper controller unit receives one 17-byte packet of controller unit ID at the head and 16 position data of pipe from $\mathrm{PC}$, then the upper controller unit can open and close a specific channel between PC and the lower controller unit corresponding to the controller ID. Subsequently, the lower controller unit receives the packet from $\mathrm{PC}$ through upper controller unit, 


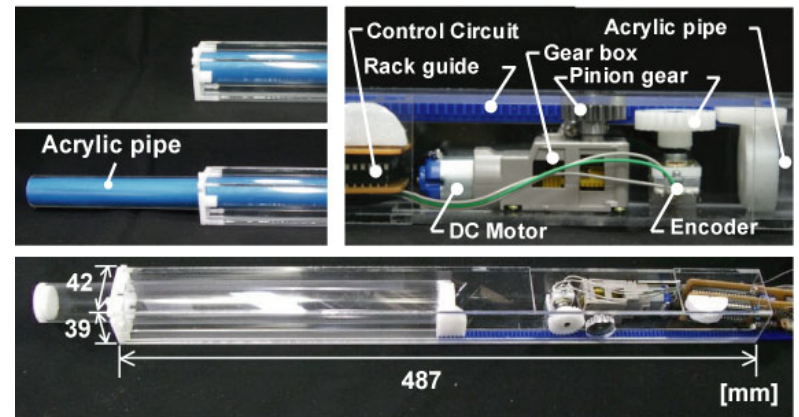

Figure 5. Cylinder module

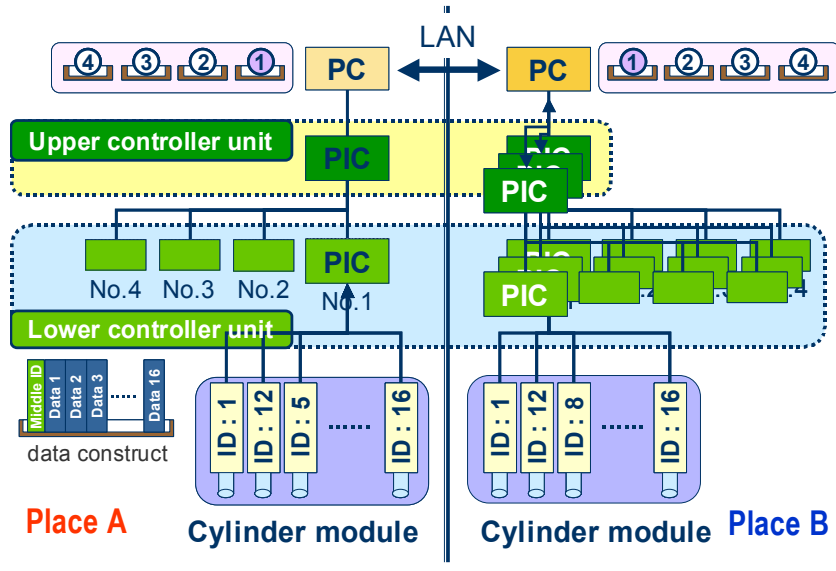

Figure 6. data communication

and the lower controller unit sends 1 byte position data to each corresponding cylinder module. Simultaneously, in up stream from module to $\mathrm{PC}$, each lower controller unit packages controller ID and 16 position data of pipe within one packet, and sends to PC through upper controller unit. This means sending and receiving process between $\mathrm{PC}$ and one lower controller unit occurs whenever a channel between PC and the lower controller unit opens. This architecture can lead that cylinder module can be available under any lower controller unit as far as each module ID isn't duplicated within one controller unit, and that communication among PC and hundreds of cylinder modules is possible by increasing upper and lower controller units in parallel.

\section{Measurement of spatial figure}

In order to measure a three-dimensional shape of remote participants, two mutually perpendicular CCD cameras are installed in front of system. Each camera captures the participant from the top and the side view. Each captured video is binarized and divided into rectangles, which corresponds to each cylinder module as shown in Figure 7. Length of detected part within each rectangle area is calculated. Each length of detected object corresponds to moving distance of each pipe. Every data, chosen as the smaller distance by comparing length calculated within rectangle in top view image and within that in side view image, is stored in data matrix.

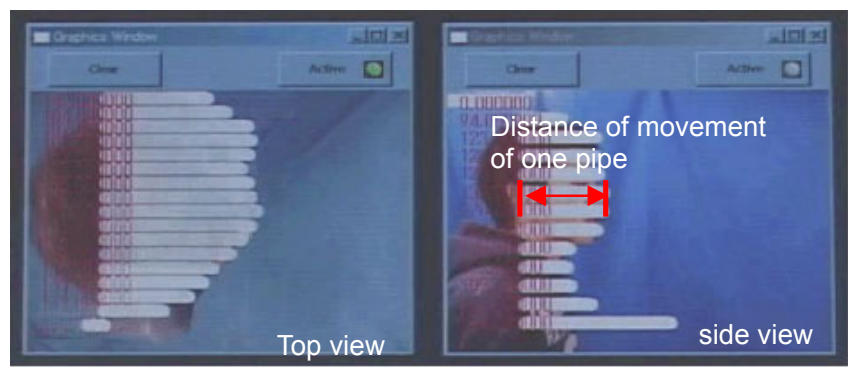

Figure 7. Measurement of a three-dimensional shape

Displaying mode and haptic interaction mode

Figure 8 shows a diagram of "ISCW" system, and Figure 9 shows appearance of "ISCW" system. We explain data processing in displaying mode and haptic interaction mode in this system. Two modes can change automatically depending on distance between participant and "wall". In displaying mode, a local PC receives data matrix of a three-dimensional shape of remote participant from remote PC via LAN, then the local PC makes packets with lower controller unit ID and 16 position data of pipe from data matrix. After that, the local PC sends the packets to each lower controller unit through upper controller unit, and then the lower controller unit sends each position data to cylinder module. Each cylinder module controls movements of pipe, so that a threedimensional shape appears on the wall. Simultaneously, video of remote place including remote participant is transmitted from remote camera, and is projected onto the wall.

In haptic interaction mode, position data of pipe should be transmitted to remote corresponding cylinder module with each other. Each lower controller unit packages its controller ID and 16 position data of pipe within one packet, and sends to PC through upper controller unit. PC sums up packets from all lower controllers into data matrix, and sends it to remote $\mathrm{PC}$ via LAN. The remote PC divides the data matrix into each packet for controller unit, and transmits to the lower controller unit. Then the lower controller unit sends position data to each cylinder module. Each cylinder module controls movements of pipe, so that position of pipe can correspond to that of remote cylinder module.

We construct "ISCW" system composed of 96 cylinder modules, and connect with another system via LAN. The results of performance test on cylinder module show delay time $0.36[\mathrm{~s}]$, settling time $0.53[\mathrm{~s}]$ (when one pipe moves from 0 up to $250[\mathrm{~mm}])$. Then data updating rate in each cylinder module within 96 cylinder modules indicates approximately $100[\mathrm{~Hz}]$. It indicates our display is available to represent spatial movements of remote partners in approximately real time.

Figure 10 shows a scene of displaying mode, and Figure 11 shows a scene of haptic interaction mode. In displaying mode, our system requires three-dimensional data in detail and adjustment of controlling movement of 


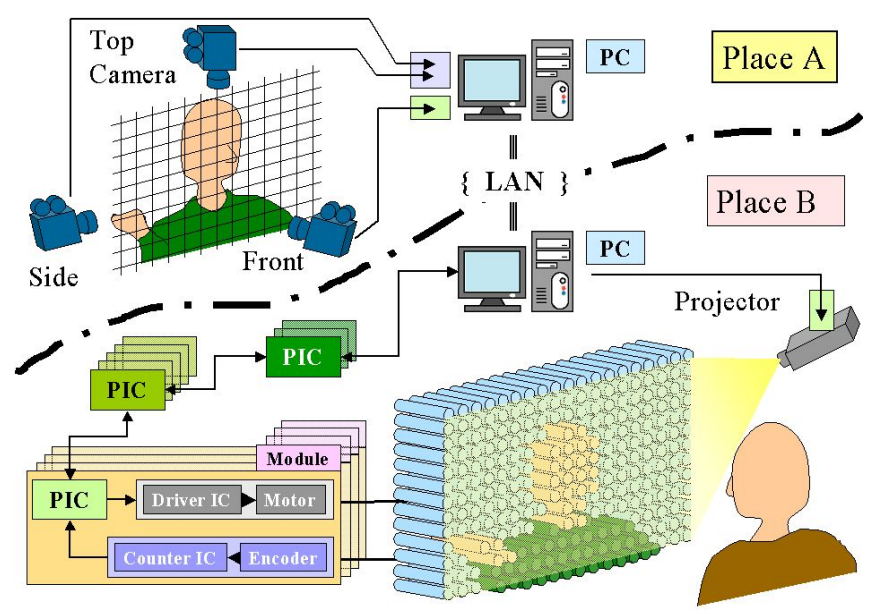

Figure 8. "ISCW" communication system configuration

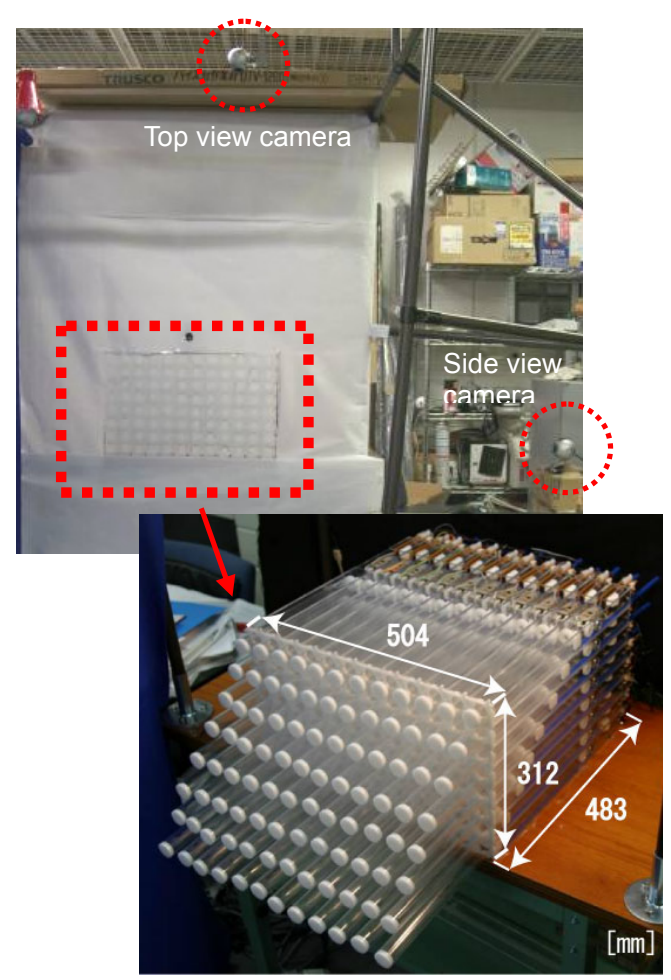

Figure 9. "ISCW" communication system

pipe to show much more precise three-dimensional appearance. In haptic interaction mode, we can pull and push any pipe at the same time.

At the last, Figure 12 shows full-scale "ISCW" system composed of 192 cylinder modules $(460 \times 680[\mathrm{~mm}])$. Three-dimensional figure based on data generated from PC appears on the wall.

\section{DISCUSSION}

Few challenging systems have been proposed and developed in order to create a virtual co-existing space. In the view of supporting a sense of interconnectedness

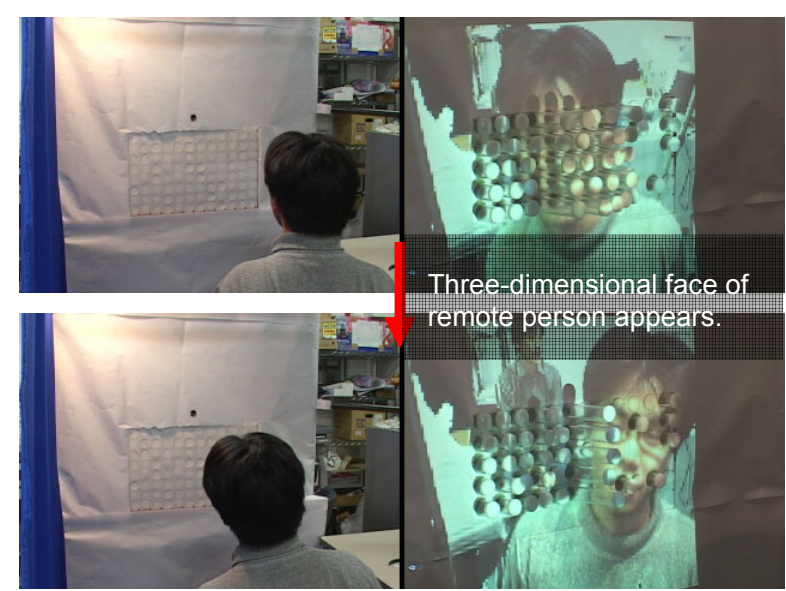

Figure 10. Displaying mode in "ISCW" system

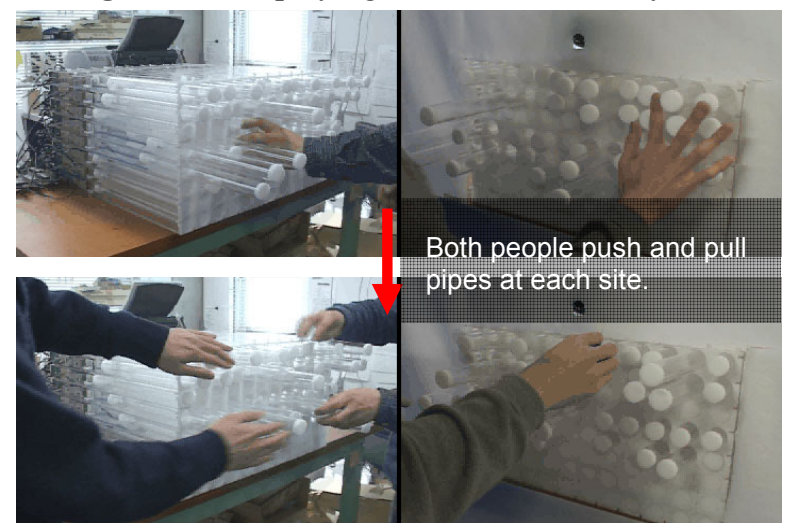

Figure 11. Haptic interaction mode in "ISCW" system
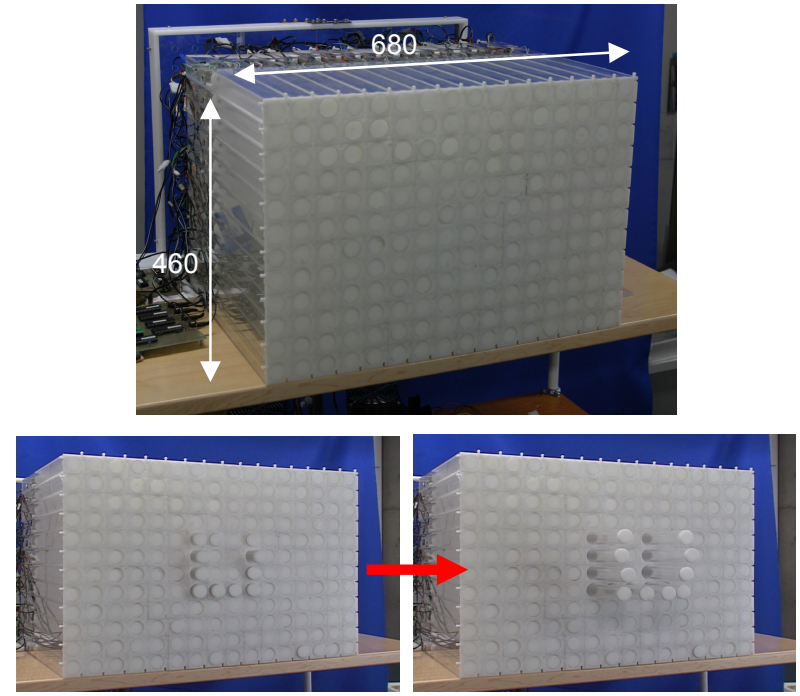

Figure 12. Full-scale "ISCW" communication system

between remote partner and self, VirtualActor [14] can create a shared virtual environment where virtual reflections of remote others and self appear and act corresponding to actions of real person. Then HyperMirror 
[15] uses a metaphor of mirror and can create a shared video space by synthesizing reversed self reflection into a video of remote place. Additionally, our previous work "Lazy Susan" communication systems propose an approach integrating not only video interaction but also tangible interaction, and can enhance interconnectedness between remote participant and local physical place and enhance a sense of presence of the remote one more than in other systems.

On the other hand, we consider robot mediated communication (RMC) system, which has attracted attention in the past several years, is another interesting way to create a virtual co-existing space in remote physical space, because robot gives us certainly its presence in face-to-face situation, and this means robot can have a possibility to express the presence of remote participant as substitute at each site respectively. However, previous RMC systems focused on mainly "mobility" only in "one" physical place. For example, an idea of teleexistence through master-slave robot system was proposed a decade ago [16]. Gestureman [17] can support a spatial indication such as explanation on machine operation from remote person. Then a health care service has just started via a robot avatar controlled by a remote medical doctor [18]. On the other hand, we have paid attention on supporting bodily presence "mutually" at both sites in our "ISCW" system. The effect of presence should be investigated furthermore; however, our system will have an availability to express multiple participants and is promising to support intergroup communication with supporting bodily presence.

\section{Summary and Conclusion}

A significance of a sense of co-existence and connectedness among remote participants has increased in computer-mediated communication. Therefore it is required that a design approach to create a co-existing space at which remote participants are bodily present. As a result, we have proposed a design method of integrating representing reflections of communication partner and self, and interacting with physical object that is linked with corresponding remote one in order to support bodily presence so far. We develop this idea and devise "Interactive Spatial Copy Wall" system serving as a tangible human avatar by representing a three-dimensional appearance of remote participant and supporting haptic interaction with remote participant with hundreds of movable pipes. We explain this idea and design process from mockup in this paper. Then we implemented "ISCW" system based on modularity. One cylinder module is the smallest element of "ISCW" system, and we can construct "ISCW" system integrating these modules. At this present, we construct networked "ISCW" system composed of 96 cylinder modules and standalone fullscale system of 192 modules. At the next step, we'll investigate an availability of our system to create a virtual co-existing space.

\section{Acknowledgements}

This research is supported partially by a Grant-in-Aid for the WABOT-HOUSE Project by Gifu Prefecture. The authors would like to thank undergraduate students Midori Sakaguchi, Teruyoshi Miyazaki and Takashi Uchiyama for their helps.

\section{References}

[1] Gutwin, C., and Greenberg, S. : The Importance of Awareness for Team Cognition in Distributed Collaboration; Team Cognition: Understanding the Factors that Drive Process and Performance, APA Press, pp. 177-201 (2004).

[2] Hubert L. Dreyfus : On the Internet (Thinking in Action); Routledge (2001).

[3] Fish,R., Kraut,R., Chalfonte,B. :The VideoWindow System in Informal Communications; Proc.of CSCW'90, pp.1-11 (1990).

[4] Shimizu, H., Kume, T., Miwa, Y., Miyake, Y.: Ba and Cocreation [in Japanese]; NTT Publishing Co. (2000).

[5] Shimizu, H: The Thoughts of "Ba": Creative Stages of Life [in Japanese]; University of Tokyo Press (2003).

[6] Kuwabara, K., et al.: Connectedness Oriented Communication: Fostering a Sense of Connectedness to Augment Social Relationships, IPSJ Journal, Vol. 43, No. 11, pp.3270-3279(2002).

[7] http://www.mimamori.net/index.html

[8] Wesugi,S., Miwa,Y.: "Dual" embodied interaction for creating a virtual co-existing space; The 6th Annual International Workshop on Presence, Presence2003, www.presence-research.org/p2003.html (2003).

[9] Wesugi,S., Ishikawa,K., Katayama,T., and Miwa,Y.: Representations of bodily interaction on networked "Lazy Susan"; Proc. of CIRA2003, ,pp.223-228(2003)

[10] Sellen, A.: Speech patterns in video-mediated conversations; Proc. of CHI '92, ACM Press, pp.49-59, (1992).

[11] Ishii, H., Kobayashi, M. Grudin, J., Integration of Interpersonal Space and Shared Workspace: ClearBoard Design and Experiments; ACM Transactions, ACM Press, Vol. 11, No. 4. pp. 349-375 (1993).

[12] Tang J. C., Minneman, S. L.: "VideoWhiteboard: video shadows to support remote collaboration", Proc. Of CHI'91, pp.315-322 (1991)

[13] Yamashita,J.,Kuzuoka,H.,Yamazaki,K.,Yamazak,A.: Agora: Supporting Multi-participant Telecollaboration; Proc. of HCII '99, Vol.2, pp. 543-547 (1999).

[14] Watanabe,T., Okubo,M. : Virtual Face-to-Face Communication System for Human Interaction Analysis by Synthesis, Proc. of HCII '99, pp. 182 - 186(1999).

[15] Morikawa,O., Maesako,T.: HyperMirror Toward Pleas-antto-use Video Mediated Communication System; Proc. of CSCW'98, pp.149-158 (1998).

[16] Tachi.,S.: TELE-EXISTENCE -Toward Virtual Existence in Real and/or Virtual Worlds-;in Proc. of the ICAT1991, pp.85-94 (1991).

[17] Kuzuoka, H., Oyama, S., Yamazaki, K., Suzuki, K., Mitsuishi, M., GestureMan: A Mobile Robot that Embodies a Remote Instructor's Actions, Proc. of CSCW2000, pp.155162(2000).

[18] http://www.intouchhealth.com/index.html 